\title{
A fuzzy modeling for single machine scheduling problem with deteriorating jobs
}

\author{
Mohammad Mahavi Mazdeh $^{\mathrm{a}^{*}}$, Farzad Zaerpour ${ }^{\mathrm{a}}$ and Farzad Firouzi Jahantigh \\ ${ }^{a}$ Department of Industrial Engineering, Iran University of Science and Technology, Narmak, Tehran Iran \\ ${ }^{b}$ Department of Industrial Engineering, University of Sistan \& Blochestan, Zahedan, Iran

\begin{tabular}{|c|c|}
\hline ART I C L E I N F O & A B S T R A C T \\
\hline $\begin{array}{l}\text { Article history: } \\
\text { Received } 1 \text { January } 2010 \\
\text { Received in revised form } \\
10 \text { May } 2010 \\
\text { Accepted } 1 \text { June } 2010 \\
\text { Available online 2 June } 2010 \\
\text { Keywords: } \\
\text { Scheduling, Sequencing } \\
\text { Single machine } \\
\text { Work in process }\end{array}$ & $\begin{array}{l}\text { This paper addresses a bi-criteria scheduling problem with deteriorating jobs on a single } \\
\text { machine. We develop a model for a single machine bi-criteria scheduling problem (SMBSP) } \\
\text { with the aim of minimizing total tardiness and work in process (WIP) costs. WIP cost increases } \\
\text { as a job passes through a series of stages in the production process. Due to the uncertainty } \\
\text { involved in real-world scheduling problems, it is sometimes unrealistic or even impossible to } \\
\text { acquire exact input data. Hence, we consider the SMBSP under the hypothesis of fuzzy L-R } \\
\text { processing time's knowledge and fuzzy L-R due date. The effectiveness of the proposed model } \\
\text { and the denoted methodology is demonstrated through a test problem. }\end{array}$ \\
\hline
\end{tabular}

Job deterioration

Bi-objective

Fuzzy decision making

(C) 2010 Growing Science Ltd. All rights reserved.

\section{Introduction}

Work in process (WIP) plays an important role on total production cost for many industries and the cost of WIP increases as more labor, machine hour and materials are added to production process. During the past few years, there has been growing interest on proposing new methods for handling WIP. According to Yang (2009), any scheduling problem, with the objective of minimizing total completion time, minimizes the average WIP inventory during the entire manufacturing process of jobs. In this case, the WIP cost of a job remains the same throughout the manufacturing process. They considered two new machine flow shop scheduling problems where the objective is to minimize the total WIP and then a heuristic is introduced for a class of problem instances. González et al. (2008) proposed a multi-objective WIP model. Their model is based on Simulation-Optimization modeling and Pareto Front concepts. Papadopoulos and Vidalis (2001) studied minimizing the average WIP inventory, subject to a minimum required throughput. They also proposed a heuristic algorithm to reduce the search space. Tsourveloudis et al. (2000) considered three fuzzy control modules to keep the WIP inventory at low levels, along with high machine utilization. Gupta et al. (2001) showed that there are situations in which increased preventive maintenance activity can lower WIP. They also studied the interaction between the duration of preventive maintenance activity and switchover times. Karsak and Kuzgunkaya (2002) proposed a fuzzy multiple objectives programming to determine FMS alternative through maximizing various objectives such as reduction in labor cost, reduction in setup cost and reduction in WIP. Tsourveloudis (2010) showed that sufficiently of production system's performance cannot be represented by WIP itself. Also the accumulated orders backlog is an

* Corresponding author. Fax: +98-21-7724-0482

E-mail addresses: mazdeh@iust.ac.ir (M. Mahdavi Mazdeh) 
important factor and when demand is relatively high, other factors such as service rate and consequently backlog are more important than WIP. In recent years, there has been significant interest in scheduling problems with deteriorating jobs. Jobs may deteriorate while waiting to be processed and job processing times can be defined as functions of their starting times and positions in the sequence. For instance, any reduction in the temperature of an ingot, while waiting to enter the rolling machine, needs the ingot to be reheated before rolling or the time required to control a fire will be increased, if there is a delay in the fire-fighting efforts. Deteriorating job scheduling problem was first introduced by Browne and yechiali (1990). Since then, deteriorating job scheduling problems have been widely discussed. Wang and Xia (2005) considered the scheduling problems under decreasing linear deterioration. They proved that an optimal schedule could be determined by Johnson's rule for two-machine flowshop scheduling problem to minimize the makespan. Jeng and Lin (2005) studied a scheduling problem of minimizing the total completion time on a single machine where the processing time of a job is a setup function of its starting time and a due date that is common to all jobs. They showed that this problem is NP-hard and proposed a lower bound along with some elimination rules to design a branch-and-bound algorithm to derive optimal solutions from a practical aspect. Wang (2007) introduced the single-machine scheduling problem with the effects of learning and deterioration. He showed that even in the presence of learning effect and deteriorating jobs, still single-machine makespan and sum of completion times minimization problems remain polynomially solvable by the SPT rule. Xu et al. (2008) investigated a parallel machine scheduling problem with almost periodic maintenance activities in which they proposed a $2 T^{\prime} / T$-approximation algorithm to minimize the makespan. Oron (2008) considered a single machine scheduling problem with simple linear deterioration. He studied many properties on the structure of an optimal schedule, and introduced two efficient heuristics to solve the problem. Roy et al. (2007) proposed an inventory model for a deteriorating item with stock dependent demand. They considered a fuzzy deterioration rate and suggested a solution methodology. According to literature, minimizing the WIP cost and the scheduling problem with the objective of minimizing total completion time in the single machine are the same, and the difference arise only when the number of machines are more than one. However, in a deteriorating system, as job processing times are defined by functions of their starting times and positions in the sequence and minimizing, WIP cost could be introduced as an important objective for a single machine problem.

In this paper a bi-criteria scheduling problem with deteriorating jobs on a single machine is considered. A model for the single machine bi-criteria scheduling problem (SMBSP) is developed with the aim of minimizing total tardiness and WIP cost. To the best of our knowledge there is not any previously published work to focus on the minimization of the total tardiness and WIP cost. The rest of the paper is organized as follows. In section 2, the problem is first described, and then a multiobjectives integer programming model, with the aim of minimizing total tardiness and minimizing WIP cost is proposed. We consider the SMBSP under the hypothesis of fuzzy L-R processing time's knowledge and fuzzy L-R due date in section 3. Section 4 presents the proposed method through a hypothetical example and the conclusion of this research is summarized in section 5 .

\section{Problem description}

Consider a scheduling problem where there are $N$ independent jobs, $J_{1}, J_{2}, \ldots, J_{n}$ to be processed on single machine. Each job, $J_{i}$, has a fuzzy fixed processing time, $\widetilde{a}_{j}$, and a fuzzy due date $\widetilde{d}_{j}$ and it is deteriorated while waiting to be processed. By the effect of job deterioration, job processing time increases if it is processed with delay. All jobs are available at time zero, but machine can process only one job at a time and other jobs must wait to begin their processes. The primary purpose of this

paper is to propose a multi-objective integer programming model where the objectives are scheduling 
jobs on a single machine to minimize total tardiness and WIP cost. Next, the necessary notation and other assumptions for the proposed mathematical formulation are explained.

\section{Assumptions:}

- Job processing time is described as a function of the starting time and fixed part of the processing time of the job.

- The growth rate of the processing time is independent of the machine.

- The jobs are available at time zero.

- The jobs are independent of each other.

- No job preemption is allowed.

\section{Known parameters}

$N \quad$ total number of jobs to be scheduled

$i, j \in I=\{0,1, \ldots, N\}$ designate the job, where job 0 is a dummy one which is always at the first sequence position

$\tilde{d}_{i} \quad$ fuzzy due date of job $i$

St ${ }_{j} \quad$ starting time of job $j, j \in I=\{0,1, \ldots, N\}$

$p_{j} \quad$ processing time of job $j, j \in I=\{0,1, \ldots, N\}$

$C_{i} \quad$ completion time of job $i$

$\tilde{a}_{j} \quad$ fuzzy fixed part of the processing time of job $j$

$\tilde{b}_{j} \quad$ fuzzy growth rate of the processing time of job $j$

$\mathrm{W}_{\mathrm{s}} \quad$ WIP cost for sequence $s$

$w_{1} \quad$ WIP cost before being processed by machine (holding cost)

$w_{2} \quad$ WIP cost while being processed by machine (added components, labor)

M a large positive number

\section{Decision variables}

$x_{i j}= \begin{cases}1 & \text { If job } j \text { immediately follows job } i \text { in sequence } \\ 0 & \text { otherwise }\end{cases}$ 


\section{Fuzzy Mathematical Model:}

$$
\begin{aligned}
& Z_{1}=\min \sum_{i=1}^{N} T(i) \\
& Z_{2}=\min \sum_{j=1}^{N} w_{1} S t_{j}+w_{2} p_{j}
\end{aligned}
$$

subject to

$$
\begin{array}{ll}
\sum_{i=0}^{N} x_{i j}=1, & j=1, \cdots N, i \neq j \\
\sum_{j=1}^{N} x_{i-1}=1, & i=1, \cdots N, i \neq j+ \\
\sum_{i=1}^{N} x_{i 0}=0, & \\
S t_{j}+M\left(1-x_{i j}\right) \geq C(i), & j=1, \cdots N \\
P_{j}=\widetilde{a}_{j}+\widetilde{b}_{j} S t_{j}, & j=1, \cdots N \\
C_{j} \geq S t_{j}+P_{j}, & j=1, \cdots N \\
T(i) \geq C_{i}-\widetilde{d}_{i}, & i=1, \cdots N \\
S t_{0}=0, C_{0}=0, C_{i,} S t_{j}, T(i), P_{j} \geq 0 . & x_{i j} \in\{0,1\}
\end{array}
$$

The objective functions of the proposed model are minimization of the total tardiness and WIP cost, respectively. According to Eq.(1), if $\mathrm{C}(i)-\mathrm{D}(i)>0$ then delivering job $i$ faces with some delay which causes tardiness. Otherwise, no tardiness is assumed. Eq.(2) calculates the sum of WIP costs and consists of two parts (before the jobs are processed by the machine or during waiting time or while the jobs are being processed). Eq.(3) ensures that always one job, job $i$, is assigned before job $j$. Eq. (4) states that each job (except the last job), is immediately followed by one job, $j$. Eq.(5) ensures that a dummy job, $(i=0)$, is positioned in the first position of the sequence. Eq.(6) declares that the starting time of a job $j$, is equal to the sum of the completion time of the preceding job. Eq.(7) expresses the processing time of a job in a sequence position which is described by functions of the starting time and fixed part of the processing time. Eq.(8) is associated with the processing time of a job to its start time and completion time. Eq.(9) expresses the relationship among the completion time of a job, its due date and its tardiness variable.

\section{Fuzzy Delphi Method}

The Delphi method is an iterative process to collect and distill the anonymous judgments of experts. Fuzzy Delphi method uses a series of data collection and analysis techniques interspersed with feedback. The Delphi method is well suited as a research instrument when there is incomplete knowledge about a problem. Fuzzy Delphi method which was proposed by Chang et al. (2000) is employed in this paper. Chang et al. (2000) used fuzzy statistics and the technique of conjugate gradient search to derive membership function of L-R fuzzy number (Fig.1) for the fuzzy forecasts. 
The procedure of Fuzzy Delphi method is illustrated in Appendix A. The category of the shapes of membership function derived in this paper is as follows (consider that $d^{(p)} \leq 1$ ):

$\mu^{(p)}(x)=\left\{\begin{array}{cc}1-\left(\left(b^{(p)}-x\right) /\left(b^{(p)}-a^{(p)}\right)\right)^{L^{(p)}} & a^{(p)} \leq x \leq b^{(p)} \\ 1 & b^{(p)} \leq x \leq c^{(p)} \\ 1-\left(\left(x-c^{(p)}\right) /\left(d^{(p)}-c^{(p)}\right)\right)^{R^{(p)}} & c^{(p)} \leq x \leq d^{(p)} \\ 0 & \text { otherwise, }\end{array}\right.$

where $P$ is the $P$ th survive question.

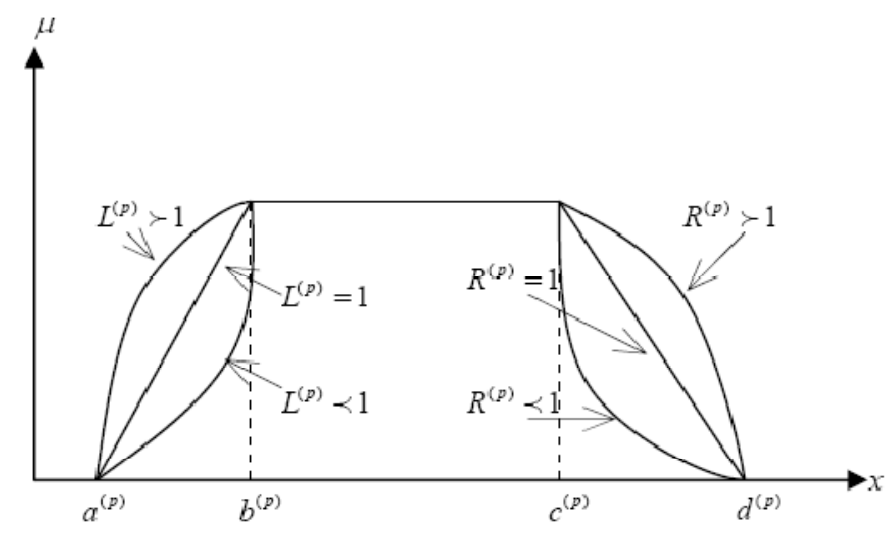

Fig 1. Membership function of L-R fuzzy number

\section{Fuzzy Defuzzification}

Yager (1981) proposed a procedure for ordering fuzzy sets based on the concept of area compensation. Area compensation possesses the properties of linearity and a ranking Index $I(\tilde{p})$ is calculated for the convex fuzzy number $\tilde{p}$ from its $\alpha$-cut, $\alpha_{\tilde{p}}=\left[P_{\alpha}^{L}, P_{\alpha}^{U}\right]$, according to the following formula,

$I(\widetilde{p})=\int_{0}^{1} \frac{1}{2}\left(p_{\alpha}^{L}+p_{\alpha}^{U}\right) d \alpha$,

Where $I(\tilde{p})$ is the center of the mean value of $\widetilde{p}$. Consider two fuzzy numbers $\widetilde{p}_{1}$ and $\widetilde{p}_{2}$; the equation $I\left(\widetilde{p}_{1}\right) \geq I\left(\widetilde{p}_{2}\right)$ implies that $\widetilde{p}_{1} \geq \widetilde{p}_{2}$ (see for example Yager (1981), Fortemps \& Roubens (1996)) which is a very simple method to apply. Yager ranking index is used as a transformation method for the membership function of the fuzzy values in the Fuzzy mathematical model. The model after defuzzification will be rewritten as follows: 
$Z_{1}=\min \sum_{i=1}^{N} T(i)$

$Z_{2}=\min \sum_{j=1}^{N} w_{1} S t_{j}+w_{2} p_{j}$

subject to

$$
\begin{array}{ll}
\sum_{i=0}^{N} x_{i j}=1, & j=1, \cdots N, i \neq j \\
\sum_{j=1}^{N} x_{i-1}=1, & i=1, \cdots N, i \neq j+1 \\
\sum_{i=1}^{N} x_{i 0}=0, & \\
S t_{j}+M\left(1-x_{i j}\right) \geq C(i), & j=1, \cdots N \\
P_{j}=I\left(\widetilde{a}_{j}\right)+I\left(\tilde{b}_{j}\right) S t_{j}, & j=1, \cdots N \\
C_{j} \geq S t_{j}+P_{j}, & j=1, \cdots N \\
T(i) \geq C_{i}-I\left(\widetilde{d}_{i}\right), & i=1, \cdots N \\
S t_{0}=0, C_{0}=0, C_{i}, S t_{j}, T(i), P_{j} \geq 0 . & x_{i j} \in\{0,1\}
\end{array}
$$

\section{Computational Experiments}

4.1 The applicable methodology for solving the F- SMBSP

We propose a methodology to solve a F- SMBSP with hypothetical data. The proposed methodology is as follow:

Step 1. Establish the fuzzy numbers by interviewing managers and employees (Tables 1-3)

\section{Table 1}

Fuzzy fixed Processing time

\begin{tabular}{cccccccccc}
\hline Job & \multicolumn{7}{c}{ Fixed Processing Time } & & \\
\hline & $\mathrm{L}$ & $\mathrm{R}$ & $\mathrm{a}$ & $\mathrm{b}$ & $\mathrm{c}$ & $\mathrm{d}$ & $\mathrm{I}$ \\
1 & 2.3 & 4.5 & 6 & 8 & 8 & 10 & 6.3 \\
2 & 5.3 & 2.1 & 3 & 4 & 4 & 5.5 & 4.3 \\
3 & 4.1 & 5.7 & 4 & 5 & 5 & 8 & 5.7 \\
4 & 1.2 & 4.3 & 6 & 7.4 & 7.4 & 9.1 & 8.2 \\
5 & 2.5 & 4.1 & 3 & 7.1 & 7.1 & 8 & 5.9 \\
\hline
\end{tabular}


Table 2

Fuzzy due date

\begin{tabular}{|c|c|c|c|c|c|c|c|}
\hline \multirow[t]{2}{*}{ Job } & \multicolumn{4}{|c|}{ Due date } & \multirow[b]{2}{*}{$\mathrm{c}$} & \multirow[b]{2}{*}{$\mathrm{d}$} & \multirow[b]{2}{*}{ I } \\
\hline & $\mathrm{L}$ & $\mathrm{R}$ & $\mathrm{a}$ & $\mathrm{b}$ & & & \\
\hline 1 & 4 & 3.2 & 7 & 10 & 10 & 12 & 7.9 \\
\hline 2 & 3 & 1 & 11 & 12 & 13 & 15 & 12.5 \\
\hline 3 & 2 & 3.5 & 12 & 13 & 13 & 15 & 13.9 \\
\hline 4 & 2.2 & 4.5 & 14 & 14.4 & 14.4 & 16 & 15.2 \\
\hline 5 & 3.5 & 4.8 & 15 & 15.1 & 15.1 & 17 & 16.3 \\
\hline
\end{tabular}

Table 3

Fuzzy growth rate of the processing time

\begin{tabular}{lllllllll}
\hline Job & \multicolumn{7}{c}{ Growth rate of the processing time } \\
\hline & $\mathrm{L}$ & $\mathrm{R}$ & $\mathrm{a}$ & $\mathrm{b}$ & $\mathrm{c}$ & $\mathrm{d}$ & $\mathrm{I}$ \\
1 & 2.4 & 4.7 & 0.2 & 0.3 & 0.3 & 0.5 & 0.41 \\
2 & 4.3 & 3.1 & 0.1 & 0.15 & 0.15 & 0.18 & 0.13 \\
3 & 4.3 & 5.8 & 0.12 & 0.13 & 0.13 & 0.17 & 0.16 \\
4 & 1.5 & 3.3 & 0.2 & 0.21 & 0.21 & 0.31 & 0.26 \\
5 & 3.5 & 2.1 & 0.14 & 0.15 & 0.15 & 0.19 & 0.16 \\
\hline
\end{tabular}

Step 2. Use Yager ranking method to defuzzify the fuzzy numbers to crisp numbers

MATLAB $\mathbb{R}$ is used to solve the algorithms of Yager ranking index of membership functions. (The last column in Tables 1-3)

Step 3. Use the LP-metric objective function (Eq.21), to consider two objective functions simultaneously,

$\min \left[\beta_{1} \frac{z_{1}-z_{1}^{*}}{z_{1}^{*}}+\beta_{2} \frac{z_{2}-z_{2}^{*}}{z_{2}^{*}}\right]$.

For this example we use $\beta_{1}=0.6, \beta_{2}=0.4, W_{1}=0.2$ and $W_{2}=0.3$.

Here, $\beta_{1}$ and $\beta_{2}$ are the weights of the objective function which are given by the decision maker. Using this LP-metric objective function and considering F-SMBSP constraints yields a single 
objective integer programming model which is solved using a simple optimization procedure. The optimal values of $z_{1}^{*}$ and $z_{2}^{*}$ for the example of this paper are 48.72 and 26.68 , respectively.

\section{Conclusion}

In this paper, a model was developed for scheduling SMBSP to minimize job tardiness and WIP cost with job deterioration. The proposed model of this paper provides a practical approach to solve realworld problems. We have also considered job deterioration as part of our modeling formulation where the job processing times are defined by a function of their starting times and positions in the sequence. The proposed model is vital as a decision support tool for many practical applications and helps to achieve a job scheduling scheme balancing the WIP cost and the job tardiness. Due to the uncertainty involved in real-world scheduling problems, fuzzy Delphi method is applied. Yager ranking method is employed to transfer the result of fuzzy Delphi method to crisp values. Further research can be focused on investigating the complexity of this problem and we leave it as an open area of research.

\section{Appendix A: Fuzzy Delphi method used in this paper (Yager (1981))}

Step 1. Set the iteration counter $k$ equal to one

Step 2. A group of $\mathrm{n}$ experts is desired to give an interval-valued opinion $\left[q_{k}^{(i)}, r_{k}^{(i)}\right]$ on each survey where $i$ is the ith expert and $p$ is the $p$ th survey question

Step 3. For each survey, item $p$ computes a discrete membership function:

(1) Let $U=[0,1]$ be the universe of discourse. Partition $U$ into $S$ contiguous interval:

$I_{1}, I_{2}, \ldots, I_{s}$.For each $I_{s}$ let $\chi_{s}$ denotes the midrange of $I_{s}$ to represent $I_{s}$.

(2) Calculate $y_{s}^{(p)}$ as follow:

$y_{s}^{(p)}=\sum_{i=1}^{n} \delta_{s}^{(i, p)}$,

where

$\delta_{s}^{(i, p)}= \begin{cases}1 & \text { if } x s \in\left[q_{k}^{(i)}, r_{k}^{(i)}\right]^{(p)} \\ 0 & \text { otherwise. }\end{cases}$

(3) The discrete membership function for survey $p$ then can be characterized by

$Y^{(p)}\left(X_{s}\right)=\frac{y_{s}^{(p)}}{y_{*}^{(p)}}, \quad s=1, \ldots, S$.

where

$y_{*}^{(p)}=\max _{s=1, \ldots, S}\left\{y_{s}^{(p)}\right\}$.

Step 4. For each survey $p$, use the results obtained in Step 3 and the technique of the conjugate gradient search to obtain the continuous mathematically explicit membership function. The fuzzy 
opinion for the survey can be conveniently denoted as $O_{k}^{(p)}=\left(a_{k}^{(p)}, b_{k}^{(p)}, c_{k}^{(p)}, d_{k}^{(p)}\right)$ with $a_{k}^{(p)} \leq b_{k}^{(p)} \leq c_{k}^{(p)} \leq d_{k}^{(p)}$.

Step 5. Generate the feedback information for the next iteration by using the $\alpha$-level cut of fuzzy opinion $O_{k}^{(p)}$ :

$\left[O_{k}^{(p)}\right]_{\alpha}=\left\{x \mid \mu_{k}^{(p)}(x) \geq \alpha\right\}=\left\{l_{k}^{(p)}(x), u_{k}^{(p)}(x)\right\}$.

Step 6. Prepare data for stability testing of fuzzy Delphi process, by using the OM index for each item $p$ :

$O M\left(O_{k}^{(p)}\right)=\int_{\rho^{*}}^{1} \varpi(w)\left[x_{1}(w) \times l_{k}^{(p)}(w)+x_{2}(w) \times u_{k}^{(p)}(w)\right] d w$,

where $\varpi(w)$ denotes the weighting measure of the $w$-level cuts of $O_{k}^{(p)}$ and $x_{1}(w)$ and $x_{2}(w)$ the measures of pessimism an optimism, respectively, of $O_{k}^{(p)}$ under $w$ and also $\rho^{*}$ assumed to be greater than zero.

Step 7. If all the differences of the fuzzy opinions between two consecutive iterations for each item $p$ appear to be smaller than the given criterion $\delta$, the process is complete and the final estimates are the fuzzy opinions obtained at the last iteration; Otherwise increase $k$ by one and return to Step 2.

\section{References}

Browne, S. \& Yechiali, U. (1990). Scheduling deteriorating jobs on a single processor, Operations Research, 38, 495-8.

Chang, P. T., Huang, L. C. \& Lin, H. J. (2000). The fuzzy Delphi via fuzzy statistics and membership function fitting and an application to human resources, Fuzzy Sets and Systems, 112, 511-520.

Fortemps, P. \& Roubens, M. (1996). Ranking and defuzzification methods based on area compensation, Fuzzy Sets System, 82, 319-330.

González, V., Alarcón, L. F. \& Molenaar, K. (2008). Multiobjective design of Work-In-Process buffer for scheduling repetitive building projects, 95-108 Automation in Construction.

Gupta, D., Gunalay, Y. \& Srinivasan, M .M. (2001). The relation between preventive maintenance and manufacturing system performance, European Journal of Operation Research, 132, 146162.

Jeng, A. \& Lin, B. (2005). Minimizing the total completion time in single-machine scheduling with step-deteriorating job, Computers \& Operations Research, 32, 521-536.

Karsak, E. E. \& Kuzgunkaya, O. (2002). A fuzzy multiple objective programming approach for the selection of a flexible manufacturing system, Int. J. Production Economics, 71, 101-111.

Lingo User's Guide. (1999). LINDO Systems Inc., Chicago.

Oron, D. (2008). Single machine scheduling with simple linear deterioration to minimize total absolute deviation of completion times. Computers \& Operations Research, 35, $2071-2078$.

Papadopoulos, H. T. \& Vidalis, M. I. (2001). Minimizing WIPinventory in reliable production lines, Int. J. Production Economics, 70, 185-197.

Roy, A., Maiti, M. K., Kar, S. \& Maiti, M. (2007). Two storage inventory model with fuzzy deterioration over a random planning horizon, Mathematical and Computer Modelling, 46 , 1419-1433.

Tsourveloudis, N. (2010). On the evolutionary-fuzzy control of WIP in manufacturing systems. Neurocomputing, 73, 648-654. 
Tsourveloudis, N. C., Dretoulakis, E. \& Ioannidis, S. (2000). Fuzzy work-in-process inventory control of unreliable manufacturing systems, Information Sciences, 127, 69-83

Wang, J. (2007). Single-machine scheduling problems with the effects of learning and deterioration, Omega, 35, $397-402$.

Wang, J. \& Xia, Z. (2005). Scheduling jobs under decreasing linear deterioration, Information Processing Letters, 94, 63-69.

Xu, D., Sun, K. \& Li, H. (2008). Parallel machine scheduling with almost periodic maintenance and non-preemptive jobs to minimize makespan, Computers \& Operations Research, 35, 1344 1349.

Yager, R. R. (1981). A procedure for ordering fuzzy subsets of the unit interval, Inform, 24, 143-161

Yang, J. (2009). Two machine flow shop scheduling problem with weighted WIP costs, Computers \& Operations Research, 36, $472-486$. 